Tecno Lógicas

ISSN 0123-7799

Vol. 18 , No. 35 , pp. $45-55$

Julio-diciembre de 2015

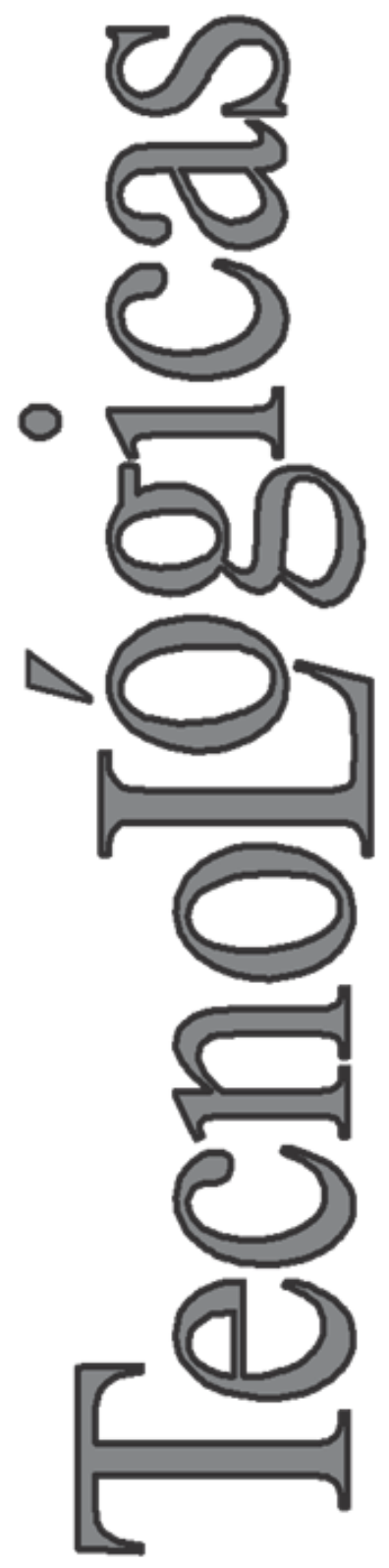

Copyright 2015 por autores y Tecno Lógicas Este trabajo está licenciado bajo una Licencia Internacional Creative Commons Atribución (CC BY)

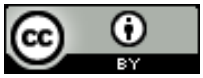

\section{Diseño, construcción y caracterización de sistema neumático para la medición de rugosidad}

\section{Design, construction and characterization of pneumatic system for measurement of roughness}

\author{
Germán A. Bacca-Bastidas ${ }^{1}$ Iván Vásquez-Valencia², \\ y Julián Pérez-Ordóñez ${ }^{3}$
}

Recibido: 2 de febrero de 2015, Aceptado: 3 de junio de 2015

Cómo citar / How to cite

G. A. Bacca-Bastidas, I. Vásquez-Valencia y J. Pérez-Ordóñez, "Diseño, construcción y caracterización de sistema neumático para la medición de rugosidad”, Tecno Lógicas, vol. 18, no. 35, pp. 45-55, 2015.

1 Ingeniero mecánico, Ph. D, Departamento de Física, Universidad del Cauca, Popayán-Colombia, gbacca@unicauca.edu.co

2 Ingeniero físico, Universidad del Cauca, Popayán-Colombia, cvasquez@unicauca.edu.co

3 Ingeniero físico, Universidad del Cauca, Popayán-Colombia, jrperez@unicauca.edu.co 


\section{Resumen}

Este artículo tiene como propósito presentar los resultados obtenidos a partir del diseño, construcción y caracterización de un amplificador neumático tobera-aleta, empleado en la medición de rugosidad de superficies planas. Para la construcción del sensor se utilizaron materiales de bajo costo y la mayoría de las piezas se obtuvieron mediante mecanizado. La adquisición de datos se realizó a través de PC utilizando como interfaz una tarjeta Arduino. El modelo matemático no-lineal del sensor está basado en las ecuaciones de flujo de gas perfecto a través de un orificio y la ley de continuidad para un volumen de control. La caracterización de parámetros físicos se obtuvo por medio de técnicas de laboratorio basadas en la respuesta transitoria que experimenta la presión de un gas en procesos de llenado y vaciado de cámaras de volumen constante, haciendo uso de herramientas computacionales para el ajuste de curvas experimentales. La validación del modelo se hizo con base en las especificaciones de respuesta transitoria que presenta un sistema dinámico frente a una entrada paso o escalón. Para la medición de la rugosidad, se empleó el modelo matemático de rugosidad promedio, $R_{a}$, y los datos medidos por el sensor fueron obtenidos en papeles de lija desde el tamaño P1000 hasta el tamaño P2000, tomando como referencia para su validación los valores de rugosidad promedio indicados por la norma FEPA.

\section{Palabras clave}

Rugosidad promedio, rugosidad superficial, volumen de control, sensor tobera-aleta, coeficiente de descarga, respuesta transitoria.

\section{Abstract}

This article aims to present the results obtained from the design, construction and characterization of a pneumatic flapper-nozzle amplifier, employed in the measurement of average surface roughness. In the construction of the sensor, low cost materials were used and most pieces were obtained by machining. The data acquisition was performed through PC, using an Arduino interface board. The nonlinear mathematical model of the sensor is based on equations of perfect gas flow through an orifice and the continuity law for a control volume. The characterization of physical parameters obtained through laboratory techniques based on the transient response of the gas pressure in the pressurization and depressurization processes of constant volume chambers, using computational tools for adjusting experimental curves. The validation of the model was based on the specifications of transient response that presents a dynamic system for a step input. For the measurement of the roughness, the mathematical model of average roughness, $R_{a}$, was used, and the measured data by the sensor were obtained in sandpapers from P1000 to P2000 size, with reference for validation values of average roughness indicated by the FEPA standard.

\section{Keywords}

Average roughness, surface roughness, volume control, nozzle-flapper sensor, discharge coefficient, transient response. 


\section{INTRODUCCIÓN}

La neumática es la rama de la potencia fluida que utiliza el aire (u otros gases) como medio para la transmisión de señales y potencia. Los sistemas neumáticos se utilizan a gran escala en la instrumentación y control de procesos industriales, así como en la automatización de maquinaria de producción.

El sensor tobera-aleta ha sido ampliamente utilizado en la medición de pequeños desplazamientos debido a su sencillo diseño, bajo consumo de energía, alta precisión, alta sensibilidad y la posibilidad de trabajar en ambientes explosivos. Entre las aplicaciones del sensor tobera-aleta se pueden destacar la detección y conteo de piezas, el posicionamiento de herramientas, la medición de nivel de líquidos, el dimensionamiento de piezas, entre otras [1].

El estudio del sensor tobera-aleta tiene hoy en día gran relevancia debido a sus ventajas y aplicaciones. Debido a su gran precisión, el amplificador tobera-aleta se ha empleado en gran medida en el diseño de sistemas de posicionamiento de servoválvulas hidráulicas y neumáticas, donde una gran parte de los trabajos de investigación están encaminados al modelado, caracterización y simulación de estos dispositivos reguladores de flujo, tal como se trata en [2]-[10]. En [11] se presenta el diseño, análisis y prueba de un servosensor neumático de desplazamiento, el cual arroja buenas características estáticas en resolución, repetibilidad y exactitud, así como una alta correspondencia entre datos teóricos y experimentales, obtenida en la validación del modelo dinámico. En [12] se presenta el modelado mecanicista y estadístico de un sensor tobera-aleta, combinando el enfoque tradicional en la elaboración del modelo mecanicista con el modelado estadístico, para lograr una adecuada validación de los datos teóricos frente a los experimentales.
Por otra parte, la medición de la rugosidad constituye un factor de gran importancia para establecer la textura ideal del acabado superficial, controlando parámetros como el grado de fricción, desgaste, resistencia a la fatiga y lubricación. En efecto, alta rugosidad no hace referencia a mala calidad de una superficie, todo depende de la exigencia que debe cumplir cada pieza manufacturada. Por consiguiente, es de gran importancia la correcta cuantificación de la rugosidad, puesto que cada vez es más creciente el uso de componentes micro-mecanizados con acabados superficiales y tolerancias dimensionales de gran precisión, empleados en industrias como la automotriz, la aeronáutica y la aeroespacial, entre otras. La medición de la rugosidad de una superficie se puede efectuar por métodos de contacto mecánico y por métodos no-destructivos tales como ópticos, capacitivos, neumáticos y ultrasónicos. Los métodos ópticos presentan ciertas ventajas respecto a los demás, tales como: mayor velocidad de repuesta, mayor precisión y fiabilidad, haciendo que estos tengan mayor demanda en la industria. Sin embargo, la principal desventaja de los métodos ópticos es su elevado costo. El empleo de un método sin contacto neumático, ofrece la posibilidad de realizar una medición de rugosidad con una considerable precisión y a un bajo costo. Los trabajos de investigación en medición de rugosidad están enfocados principalmente en el desarrollo de métodos no-destructivos a través de láser, microscopía y procesamiento de imágenes, tal como se presenta en [13][17]. En [18] se presenta un estudio reciente realizado en una tobera sónica, en el cual para flujo de gas real se muestra la variación del coeficiente de descarga, y otros parámetros como el número de Reynolds, la relación de calores específicos y el radio de curvatura de la tobera, en función de la rugosidad, obteniéndose resultados con alta correspondencia respecto a estudios anteriores. 
En este artículo, la descripción funcional, el modelado matemático y la caracterización estática del sensor, son presentados en la sección 2. En la sección 3 se muestra la validación dinámica del modelo y los resultados en la medición de rugosidad; y en la sección 4 se presentan las conclusiones.

\section{METODOLOGíA}

En la Fig. 1 se muestra el esquema del sensor tobera aleta. El cuerpo del sensor es un tubo de volumen $V$, el cual está conectado por un extremo a una fuente de suministro de presión constante $P_{s}$, y por el otro extremo a una tobera, cuyo orificio de salida tiene un grado de obstrucción que depende de la posición $x$, que adopte la aleta. Puesto que la presión interna del tubo (presión de salida) $P$, depende de los flujos de masa $G_{s}$ y $G_{n}$, por lo tanto, un cambio en el desplazamiento $x$ produce un cambio en la presión de salida $P$.

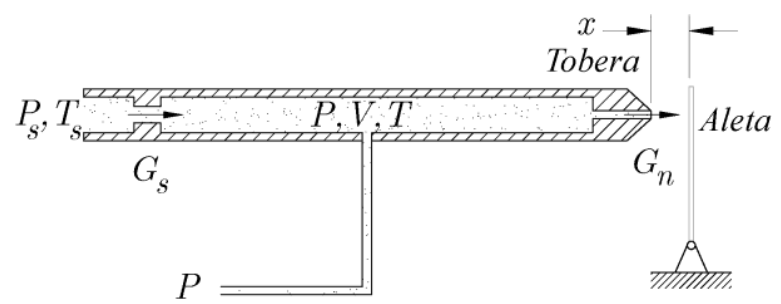

Fig. 1. Sistema tobera-aleta. Fuente: Autores

En la Fig. 2 se muestra el esquema neumático sistema tobera-aleta.

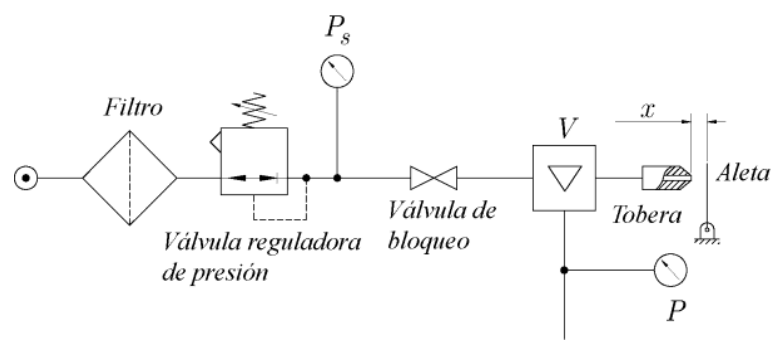

Fig. 2. Circuito neumático. Fuente: Autores

En las Fig. 3 a 5 se muestran las partes maquinadas que conforman el sistema tobera-aleta. El volumen del tubo es de
$6,48 \mathrm{~cm}^{3}$, el diámetro del orificio (chicler) es de $1 / 64 " \simeq 0,397 \mathrm{~mm}$ y el diámetro del orificio de la tobera es de $1 / 32 ” \simeq 0,794$ $\mathrm{mm}$.

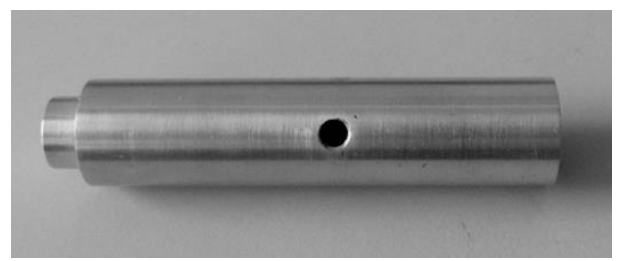

Fig. 3. Tubo de volumen $V$. Fuente: Autores

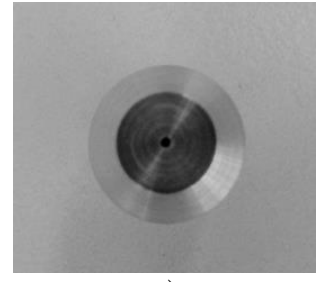

a)

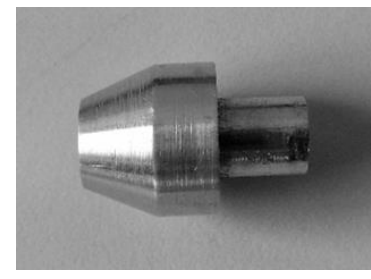

b)
Fig. 4. Tobera a) Vista superior, b) Vista lateral. Fuente: Autores

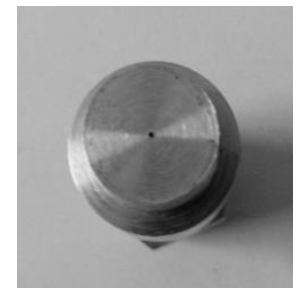

a)

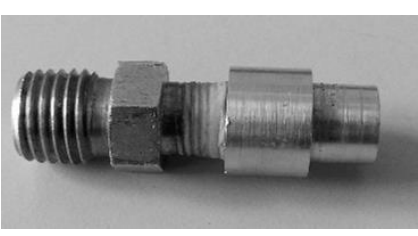

b)
Fig. 5. Orificio (chicler) a) Vista superior, b) Vista lateral. Fuente: Autores

La Fig. 6 muestra un dibujo 3D del ensamble del sensor.

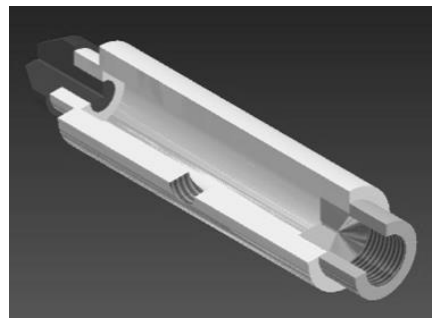

Fig. 6. Amplificador neumático tobera-aleta. Fuente: Autores

Para la obtención del modelo matemático del sensor se comienza aplicando la Ley de conservación de la masa al volumen de control $V$ (véase la Fig. 1), y considerando al aire como un gas ideal, se puede establecer: 
$G_{s}-G_{n}=\frac{V}{n R T} \frac{d P}{d t}$

donde $G_{s}$ es el flujo de masa de suministro, $G_{n}$ es el flujo de masa de salida, $n$ es la constante politrópica del proceso dentro del volumen de control, $R$ es la constante particular del aire, $T$ es la temperatura absoluta del aire dentro del volumen de control y $P$ es la presión absoluta del aire dentro del volumen de control. De (1) se obtiene:

$\frac{d P}{d t}=\frac{n R T}{V}\left(G_{s}-G_{n}\right)$

Los flujos de masa se obtienen a partir de las ecuaciones para flujo isentrópico a través de un orificio. El flujo de masa de suministro, $G_{s}$, se puede expresar como:

$G_{s}=\left\{\begin{array}{l}c_{d s} \frac{\pi D_{s}^{2}}{4} \frac{P_{s}}{\sqrt{T_{s}}} \sqrt{\frac{2 k}{(k+1) R}\left(\frac{2}{k+1}\right)^{\frac{2}{k-1}}}, \text { si } 0 \leq r_{p} \leq\left(\frac{2}{k+1}\right)^{\frac{k}{k-1}} \\ c_{d s} \frac{\pi D_{s}^{2}}{4} \frac{P_{s}}{\sqrt{T_{s}}} \sqrt{\frac{2 k}{(k-1) R}\left[r_{p}^{\frac{2}{k}}-r_{p}^{\frac{k+1}{k}}\right]}, \text { si }\left(\frac{2}{k+1}\right)^{\frac{k}{k-1}}<r_{p} \leq 1\end{array}\right.$

donde $r_{p}$ es la relación de presiones entre la presión de salida, $P$, y la presión de suministro, $P_{s}, k$ es la constante isentrópica del aire, $c_{d s}$ es el coeficiente de descarga del orificio y $D_{s}$ es el diámetro del orificio.

De igual manera, el flujo de masa de salida, $G_{n}$, se puede calcular mediante (4), donde $r_{p}=P_{0} / P$, siendo $P_{0}$ la presión atmosférica, $c_{d n}$ es el coeficiente de descarga del orificio de la tobera y $D_{n}$ es el diámetro del orificio de la tobera.

$G_{n}= \begin{cases}c_{d n} \pi D_{n} x \frac{P}{\sqrt{T}} \sqrt{\frac{2 k}{(k+1) R}\left(\frac{2}{k+1}\right)^{\frac{2}{k-1}}}, & \text { si } 0 \leq r_{p} \leq\left(\frac{2}{k+1}\right)^{\frac{k}{k-1}} \\ c_{d n} \pi D_{n} x \frac{P}{\sqrt{T}} \sqrt{\frac{2 k}{(k-1) R}\left[r_{p}^{\frac{2}{k}}-r_{p}^{\frac{k+1}{k}}\right]}, & \text { si }\left(\frac{2}{k+1}\right)^{\frac{k}{k-1}}<r_{p} \leq 1\end{cases}$

La caracterización en estado estable del sensor tobera-aleta está dada por la obtención de la curva característica presión vs. desplazamiento o función presióndesplazamiento y la obtención de los coeficientes de descarga para los orificios de entrada y salida.

\subsection{Función presión-desplazamiento}

Esta característica se determinó experimentalmente mediante el montaje de la Fig. 2, donde el desplazamiento $x$ fue regulado mediante un tornillo micrométrico. Partiendo desde $0 \mathrm{~mm}$ (orificio de la tobera completamente obturado), y variando el desplazamiento cada $0,1 \mathrm{~mm}$ hasta una distancia de $0,28 \mathrm{~mm}$, se obtuvo la curva mostrada en la Fig. 7. De la función presión-desplazamiento se puede determinar el rango de presión de salida del instrumento, el cual se encuentra entre $152 \mathrm{kPa}$ para $x=0 \mathrm{~mm}$ y $5,31 \mathrm{kPa}$ para $0,28 \mathrm{~mm}$. Además, el instrumento presenta una región de operación con un comportamiento bastante lineal en el intervalo $0,0015 \leq x \leq 0,063 \mathrm{~mm}$, para el cual $142,7 \leq P \leq 31,5 \mathrm{kPa}$, presentando en esta región una sensibilidad estática, $k_{e}$ [1], del orden de $k_{e}=1,805 \times 10^{3} \mathrm{kPa} / \mathrm{mm}$.

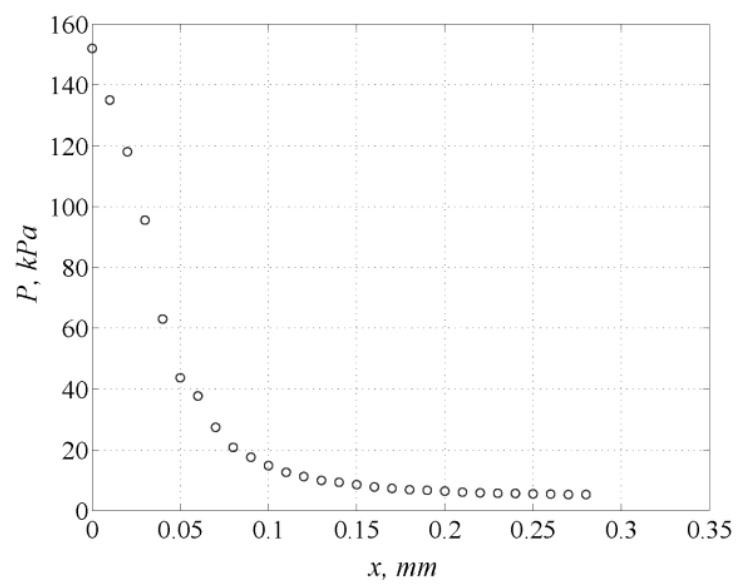

Fig. 7. Función presión-desplazamiento. Fuente: Autores

\subsection{Coeficientes de descarga}

Los coeficientes de descarga $c_{d s}$ y $c_{d n}$ se obtuvieron mediante el ajuste entre datos teóricos y datos experimentales, utilizando para ello la técnica de optimización nolineal de mínimos cuadrados. Para el coefi- 
ciente de descarga del orificio de entrada, $c_{d s}$, los datos experimentales se tomaron mediante un proceso de presurización del volumen de control, $V$, por medio de la apertura de la válvula de bloqueo, desde la presión atmosférica, $P_{0}$, hasta la presión de suministro, $P_{s}$, y manteniendo el orificio de la tobera completamente obturado. En la Fig. 8 se muestra la curva experimental de presurización para el orificio de entrada y su correspondiente curva de ajuste, cuyo coeficiente de descarga obtenido mediante la técnica de mínimos cuadrados fue $c_{d s}=0,4707$.

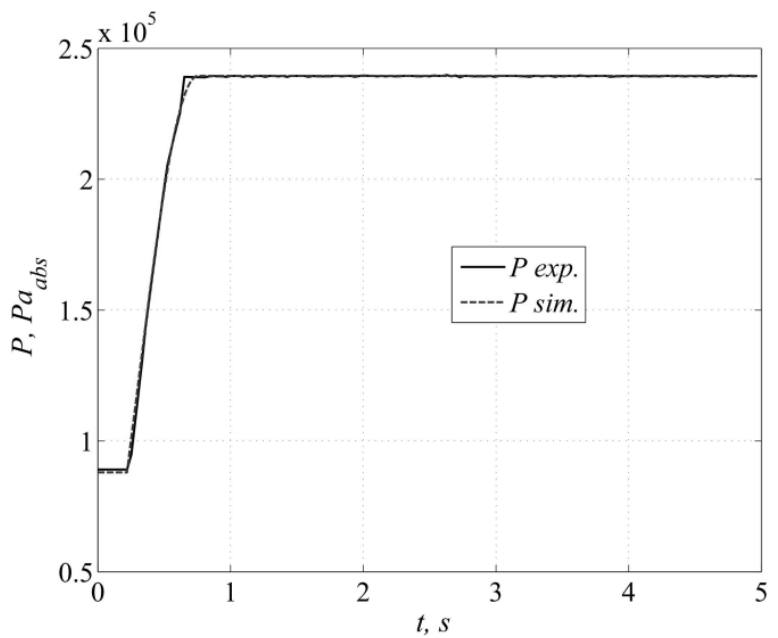

Fig. 8. Curva de presurización con $x=0 \mathrm{~mm}$. Fuente: Autores

Para el coeficiente de descarga del orificio de la tobera, $c_{d n}$, los datos experimentales fueron tomados para diferentes procesos de despresurización del volumen de control, $V$, partiendo de una presión inicial igual a la presión de suministro, $P_{s} \mathrm{y}$ abriendo el obturador para diferentes desplazamientos comprendidos en el intervalo $0,005 \leq x \leq 0,005 \mathrm{~mm}$ incrementado cada desplazamiento en 0,005 mm. En la Fig. 9 se muestra una curva característica de despresurización para $x=0,05 \mathrm{~mm}$ y su correspondiente curva de ajuste.

El comportamiento del coeficiente de descarga del orificio de la tobera, $c_{d n}$, frente al desplazamiento del obturador, $x$, se muestra en la Fig. 10. Mediante una correlación, se puede establecer un modelo para el coeficiente de descarga en término del desplazamiento del obturador como:

$$
c_{d n}=-24097 x^{3}+2204 x^{2}-50.526 x+0.48935
$$

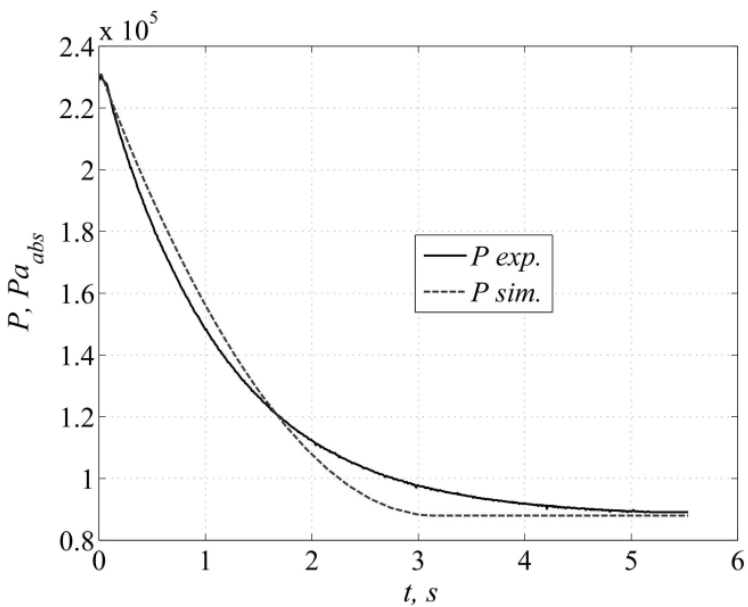

Fig. 9. Curva de despresurización con $\mathrm{x}=0,05 \mathrm{~mm}$. Fuente: Autores

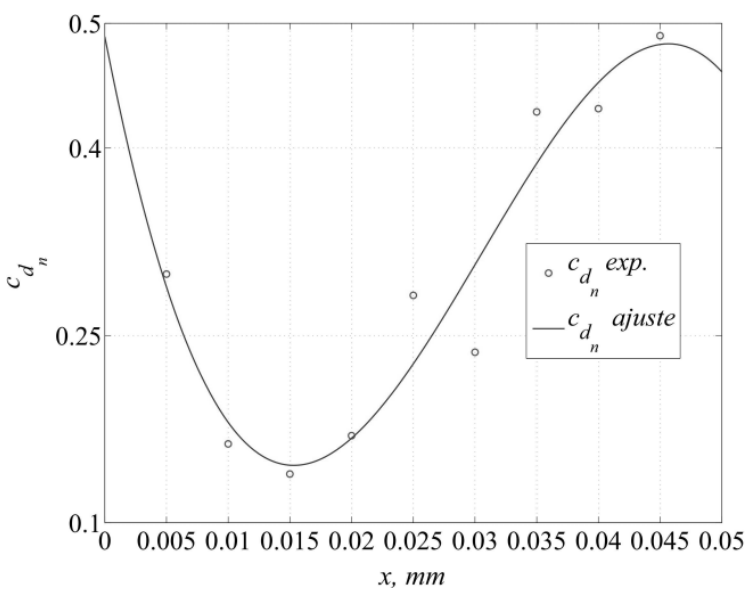

Fig. 10. Variación de $c_{d n}$ con respecto a $x$. Fuente: Autores

\subsection{Flujo de masa de suministro}

El flujo de masa de suministro, $G_{s}$, puede ser expresado mediante la relación:

$G_{s}=G\left(c_{d s}, D_{s}, P_{s}, T_{s}, P(x)\right)$

Puesto que $c_{d s}, D_{s}, P_{s}$ y $T_{s}$ son constantes, la anterior relación se reduce a:

$G_{s}=G(P(x))$

La Fig. 11 muestra la variación del flujo de masa de suministro, $G_{s}$, en función de la 
presión $P(x)$, para $x=0 \mathrm{~mm}$. Se observa que el flujo de masa de suministro presenta un comportamiento sónico (máximo), si la presión dentro del volumen de control se encuentra en el intervalo $88 \leq P \leq 126,3$ $\mathrm{kPa}$ abs, donde $P=126,3 \mathrm{kPa}$ abs (círculo) es el valor de la presión crítica $\left(P_{c}=0,528 P_{s}\right)$. Así mismo, se puede observar que el flujo de masa presenta un comportamiento subsónico en el intervalo $126,3 \leq P \leq 239,2$ $\mathrm{kPa}$ abs.

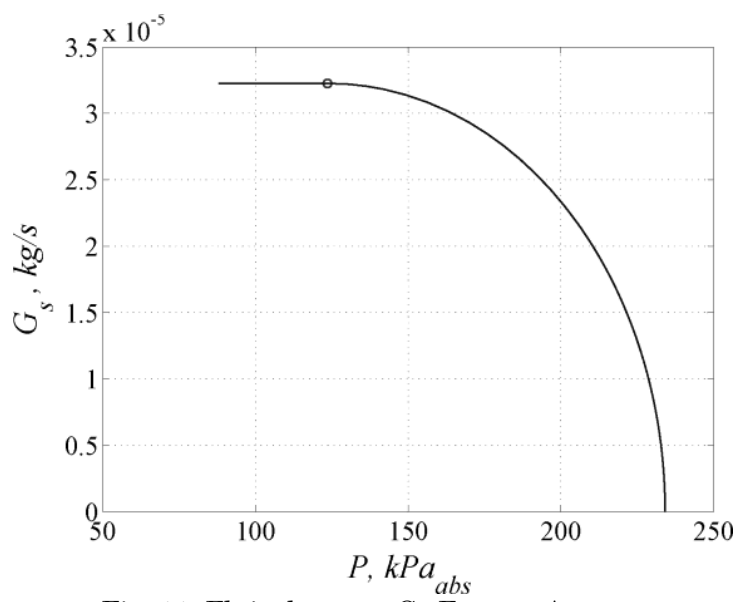

Fig. 11. Flujo de masa $G_{s}$. Fuente: Autores

En la Fig. 12 se muestra toda la posible variación del flujo de masa de suministro en función del desplazamiento, $x$, y la presión $P$. Se puede comprobar que la curva de flujo de masa de suministro, $G_{s}$, (curva a trazos y círculos), estimada a partir de la función presión-desplazamiento, $P(x)$, pertenece a la superficie de la variación de flujo masa.

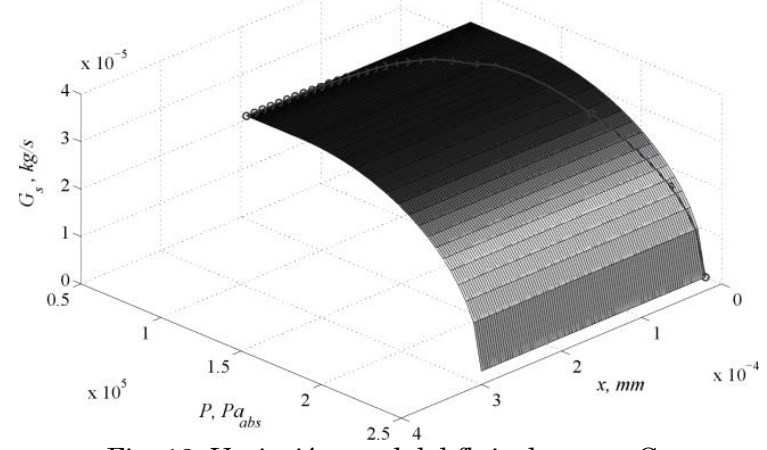

Fig. 12. Variación total del flujo de masa $G_{s}$. Fuente: Autores

\subsection{Flujo de masa de salida}

El flujo de masa de salida, $G_{n}$, se puede expresar mediante la relación:

$G_{n}=G\left(c_{d n}, D_{n}, x, P(x), T, P_{0}\right)$

Teniendo en cuenta que $c_{d n}, D_{n}, P_{0}$ y $T$ son constantes, por lo tanto:

$G_{n}=G(x, P(x))$

La Fig. 13 muestra toda la posible variación del flujo de masa de salida en función del desplazamiento y la presión. También se puede comprobar que la curva de flujo de masa de salida, $G_{n}$, (curva a trazos y círculos), obtenida a partir de la función presión-desplazamiento, $P(x)$, pertenece a la superficie de flujo de masa.

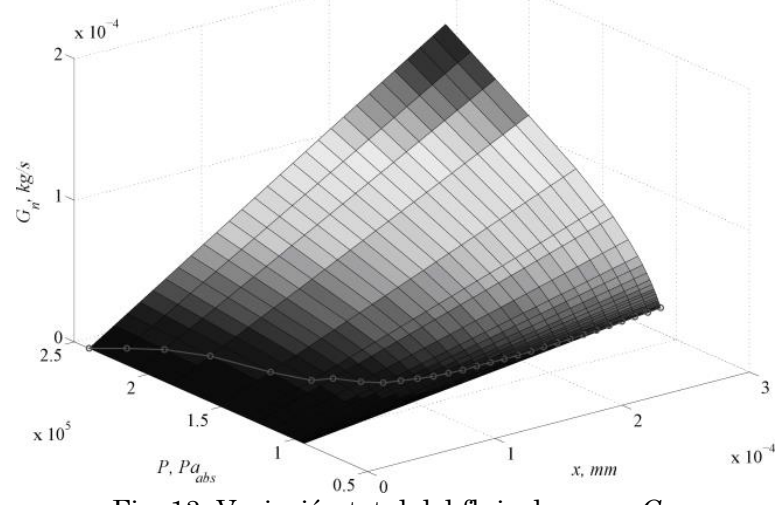

Fig. 13. Variación total del flujo de masa $G_{n}$. Fuente: Autores

\section{RESULTADOS Y DISCUSIÓN}

\subsection{Validación dinámica del modelo}

La validación dinámica del modelo se obtuvo a partir de la respuesta frente a la entrada escalón:

$$
x(t)=\left\{\begin{array}{l}
x_{i}, \text { si } t \leq 0 \\
0, \text { si } t>0
\end{array}\right.
$$


En la Fig. 14 se muestran las respuestas teórica y experimental del sensor tobera-aleta frente a la entrada escalón con $x_{i}=0,1 \mathrm{~mm}$. Se puede comprobar que los datos teóricos presentan una alta correspondencia con los datos experimentales, cabe anotar que la presencia de una especie de sobreimpulso que presentan los datos experimentales no obedece a la dinámica del sensor, sino a la dinámica de la válvula reguladora de presión que le impide mantener constante la presión de suministro. Puesto que el sensor tobera-aleta presenta una respuesta semejante a la de un sistema de primer orden [1], la constante de tiempo en base a la respuesta experimental para la cual alcanza el $63,2 \%$ de su valor final es $\tau=0,271 \mathrm{~s}$.

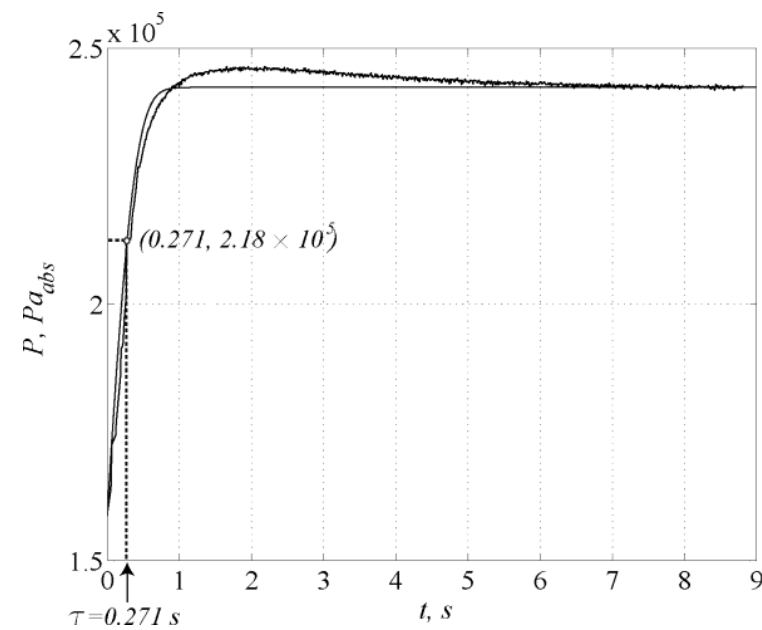

Fig. 14. Respuesta paso para $x_{i}=0,1 \mathrm{~mm}$. Fuente: Autores

En la Fig. 15 se presenta una estimación teórica del diagrama de bode del sensor tobera-aleta, a partir de su modelo matemático, de este diagrama, se puede inferir que el instrumento presenta una respuesta en frecuencia similar a la de un sistema lineal de primer orden, con una frecuencia de corte $\omega_{c}=0,2 \mathrm{~Hz}$ y un comportamiento asintótico aproximadamente de $-20 \mathrm{db} /$ década, por otra parte, se presenta un desfase de $-170^{\circ}$ a frecuencias cercanas a cero, y un comportamiento asintótico aproximado a $-300^{\circ}$ para las frecuencias altas.
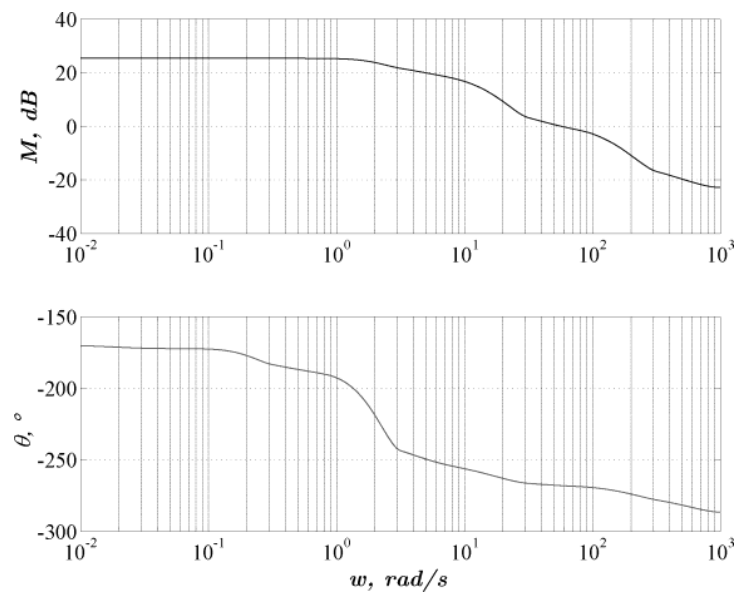

Fig. 15. Diagrama de Bode. Fuente: Autores

\subsection{Medición de la rugosidad}

\subsubsection{Montaje experimental}

La Fig. 16 muestra el montaje implementado para realizar las pruebas de medida de rugosidad promedio. El mecanismo de barrido de la muestra está accionado por un motor de corriente continua que posee una caja reductora con una relación de 9:1 de tal manera que la velocidad constante de barrido es $v=1,45 \mathrm{~mm} / \mathrm{s}$. Las muestras se colocaron a una distancia de $\mathrm{x}=0,038 \mathrm{~mm}$ que corresponde al valor medio del desplazamiento del obturador en la región activa del sensor.

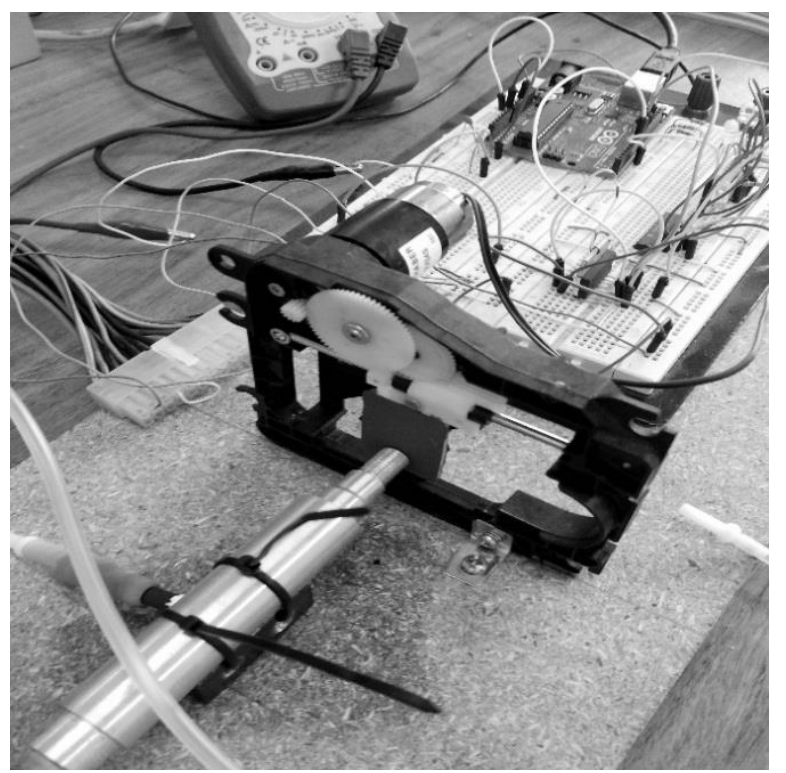

Fig. 16. Montaje experimental para medir rugosidad $R_{a}$. Fuente: Autores 


\subsubsection{Rugosidad promedio}

La rugosidad promedio, $R_{a}$, se define como la media aritmética de los valores absolutos de las desviaciones del perfil, en los límites de la longitud básica, $L$, es decir:

$R_{a}=\frac{1}{L} \int_{0}^{L}|f(z)| d z$

donde $f(z)$ es la función del perfil de rugosidad que presenta la superficie. La definición de rugosidad promedio se puede apreciar mejor en la Fig. 17.

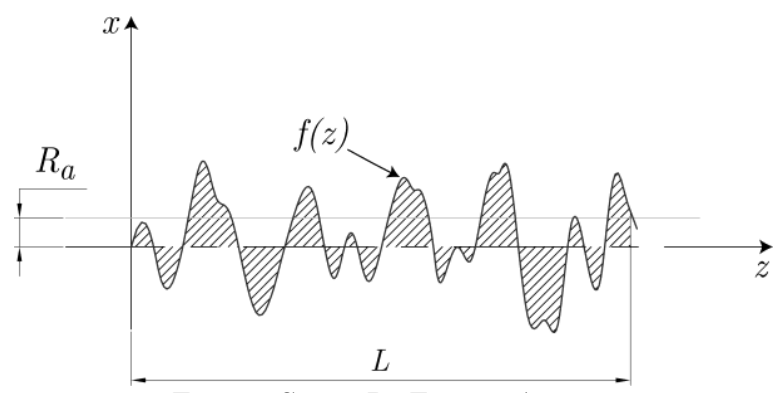

Fig. 17. Curva $R_{a}$. Fuente: Autores

La Fig. 18 muestra el perfil de rugosidad para una lija P1000, donde la rugosidad promedio, $R_{a}$, está representada por la relación entre el área bajo la curva y el desplazamiento a lo largo de la muestra.

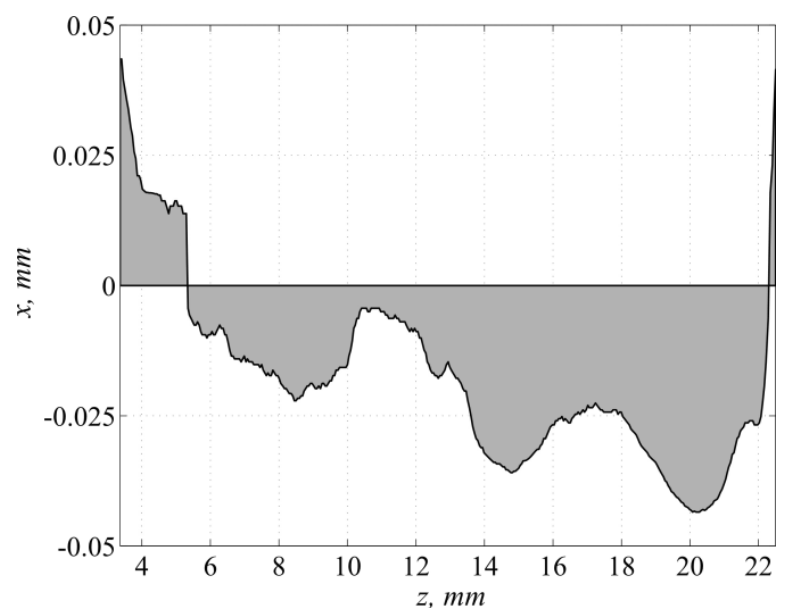

Fig. 18. Perfil de rugosidad para una lija P1000. Fuente: Autores

En la Tabla 1 se presentan los valores de rugosidad promedio para muestras de papel de lija marca Abracol, desde el tamaño de grano P1000 hasta el tamaño de grano P2000, ya que estos tamaños se encuentran dentro del rango de calibración del instrumento, el cual se encuentra entre $0,0015 \leq x \leq 0,063 \mathrm{~mm}$, que corresponde a los grados de rugosidad N7 a N12. Los valores medios de rugosidad promedio, $\bar{R}_{a}$, entregados por el sensor para muestras de diez (10) datos, $R_{a}$, fueron validados frente a los rangos de valores de rugosidad promedio estandarizados por la norma FEPA.

Tabla 1: Resultados de Rugosidad Promedio.

\begin{tabular}{cccc}
\multicolumn{5}{c}{ Fuente: Autores } \\
\hline Tamaño & \multicolumn{1}{c}{$R_{a}, \mu \mathrm{m}$} & $\bar{R}_{a}, \mu \mathrm{m}$ & $F E P A$ \\
\hline \multirow{3}{*}{ P1000 } & 18,$2 ; 19,1 ; 17,0 ; 21,2 ;$ & & 19,3 \\
& 18,$7 ; 20,5 ; 20,1 ; 17,8 ;$ & 19,18 & \\
& 20,$0 ; 19,2$ & & 17,3 \\
& 16,$3 ; 16,1 ; 14,5 ; 14,2 ;$ & & 16,3 \\
P1200 & 14,$3 ; 15,5 ; 15,8 ; 16,3 ;$ & 15,28 & \\
& 14,$8 ; 15,0$ & & 14,3 \\
& 11,$7 ; 13,5 ; 11,5 ; 13,7 ;$ & & 13,6 \\
P1500 & 12,$2 ; 10,4 ; 12,1 ; 11,2 ;$ & 12,17 & \\
& 13,$6 ; 11,8$ & & 11,6 \\
& 9,$20 ; 9,10 ; 10,2 ; 10,7 ;$ & & 11,1 \\
P2000 & 10,$9 ; 9,3 ; 10,2 ; 9,20 ;$ & 9,94 & \\
& 10,$5 ; 10,1$ & & 9,5 \\
\hline
\end{tabular}

En las cuatro pruebas de repetibilidad el sensor presentó en promedio una precisión basada en el modelo Gaussiano [1]:

$$
\Delta x= \pm k S_{x}
$$

con $k=3$, igual a $\Delta x= \pm 2,94 \mu \mathrm{m}$.

\section{CONCLUSIONES}

Con este trabajo se logró el diseño y la construcción de un sistema de medición de rugosidad promedio de una superficie, basado en el funcionamiento del amplificador neumático tobera-aleta. La construcción del sensor se caracterizó por su sencillez ya que los materiales empleados son de fácil adquisición y sus piezas fueron obtenidas por mecanizado básico. El modelo matemático empleado se ajustó perfecta- 
mente al modelo no-lineal de flujo de masa en una cámara de volumen constante con un orificio de entrada de área fija y otro de salida de área variable. La calibración estática del instrumento permitió establecer un rango de medida que varía entre $0,0015 \leq \mathrm{x} \leq 0,063 \mathrm{~mm}$, además, es importante destacar que los coeficientes de descarga de los orificios de suministro, $G_{s}, \mathrm{y}$ salida, $G_{n}$, mostraron comportamientos diferentes, manteniéndose el primero en un valor constante, mientras que el segundo presentó una variación con respecto al desplazamiento del obturador, $x$. Las curvas de flujo de masa indicaron que el consumo de aire del sensor es muy bajo, ya que el flujo crítico no supera los $3,5 \times 10^{-5}$ $\mathrm{kg} / \mathrm{s}$.

El modelo matemático presentó una alta correspondencia con los datos experimentales, hecho que se comprueba en su validación dinámica, donde se puede apreciar que el sensor presenta una alta velocidad de respuesta $(\tau=0,271 \mathrm{~s})$ y un error dinámico en estado estable tendiente a cero. El diagrama de Bode presentó el comportamiento del instrumento frente a la frecuencia similar al de un instrumento de primer orden, confirmando así el comportamiento típico de esta clase de instrumentos.

En la medición de rugosidad, se utilizó el modelo de rugosidad promedio, $R_{a}$. Realizando un barrido lineal de la superficie a medir, se observó un buen comportamiento del sensor puesto que las medidas entregadas por éste, se encuentran dentro de los rangos establecidos por la norma FEPA, confirmando así que puede ser utilizado como un sistema de medición de rugosidad no destructivo con una buena precisión y a un bajo costo.

\section{REFERENCIAS}

[1] E. Doebelin, Measurement Systems: Application and Design. McGraw-Hill, 1990.
[2] N. Z. Aung and S. Li, "A numerical study of cavitation phenomenon in a flapper-nozzle pilot stage of an electrohydraulic servo-valve with an innovative flapper shape," Energy Convers. Manag., vol. 77, pp. 31-39, Jan. 2014.

[3] N. Z. Aung, Q. Yang, M. Chen, and S. Li, "CFD analysis of flow forces and energy loss characteristics in a flapper-nozzle pilot valve with different null clearances," Energy Convers. Manag., vol. 83, pp. 284-295, Jul. 2014.

[4] S. Li, N. Z. Aung, S. Zhang, J. Cao, and X. Xue, "Experimental and numerical investigation of cavitation phenomenon in flapper-nozzle pilot stage of an electrohydraulic servo-valve," Comput. Fluids, vol. 88, pp. 590-598, Dec. 2013.

[5] Z. Peng, C. Sun, R.-B. Yuan, and P. Zhang, "The CFD analysis of main valve flow field and structural optimization for double-nozzle flapper servo valve," Procedia Eng., vol. 31, pp. 115-121, 2012.

[6] S. Li, M. M. Jacob, and S. Zhanguan, "Study of Jet Flow with Vortex and Pressure Oscillations between the Flapper-Nozzle in a Hydraulic Servo-valve," in Automation Congress (WAC), 2012, pp. 1-4.

[7] Dongjie $\mathrm{Mu}$ and Changchun Li, "A new mathematical model of twin flapper-nozzle servo valve based on input-output linearization approach," in 2011 2nd International Conference on Artificial Intelligence, Management Science and Electronic Commerce (AIMSEC), 2011, pp. 3662-3666.

[8] Z. Khodaee, M. Zareinejad, and S. S. Ghidary, "Modeling of a two-stage flapper-nozzle electrohydraulic servo valve exposed to acceleration," in 2014 Second RSI/ISM International Conference on Robotics and Mechatronics (ICRoM), 2014, pp. 268-273.

[9] Zhang Xi, Yin Yaobao, and Huang Weida, "Influence of temperature on null position pressure characteristics of flapper-nozzle servo valve," in 2010 International Conference On Computer Design and Applications, 2010, vol. 3, pp. V3-257-V3-261.

[10] B. Gang, C. Tinghai, H. Yao, G. Xiangdong, and G. Han, "A nozzle flapper electro-pneumatic proportional pressure valve driven by piezoelectric motor," in Proceedings of 2011 International Conference on Fluid Power and Mechatronics, 2011, pp. 191-195.

[11]Y. Zhang, K. Kawashiwa, T. Fujita, and T. Kagawa, "Development of an air servo displacement sensor," Precis. Eng., vol. 28, no. 4, pp. 435-442, Oct. 2004.

[12] V. Bokov, "Nozzle-flapper sensor mechanistic and statistical concurrent modeling," Measurement, vol. 37, no. 1, pp. 47-61, Jan. 2005. 
[13]Z. Yilbas and M. S. J. Hasmi, "Surface roughness measurement using an optical system,” J. Mater. Process. Technol., vol. 88, no. 1-3, pp. 10-22, Apr. 1999.

[14]X. Zhao and Z. Gao, "Surface roughness measurement using spatial-average analysis of objective speckle pattern in specular direction," Opt. Lasers Eng., vol. 47, no. 11, pp. 1307-1316, Nov. 2009.

[15] Z. Li and J. Liu, "The In-process and Real-Time Roughness Measuring System Design for FreeForm Surface," in 2010 International Conference on Computational and Information Sciences, 2010, pp. 824-826.
[16] Y. Quinsat and C. Tournier, "In situ non-contact measurements of surface roughness," Precis. Eng., vol. 36, no. 1, pp. 97-103, Jan. 2012.

[17] C.-C. Kuo, Y.-R. Chen, C.-Y. Tong, and J.-W. Lee, "A low-cost optical inspection system for rapid surface roughness measurements of $\mathrm{CrCN}$ hard films," Opt. - Int. J. Light Electron Opt., vol. 124, no. 14, pp. 1902-1906, Jul. 2013.

[18] H. Ding, C. Wang, and Y. Zhao, "Surface roughness effect on flow measurement of real gas in a critical nozzle," Measurement, vol. 68, pp. 82-91, May 2015. 